EPiC Series in Computing
Volume 69, 2020, Pages 307-316
Proceedings of 35th International Confer-
ence on Computers and Their Applications

\title{
New Monitoring Architectures for underwater oil/Gas Pipeline using Hyper sensors
}

\author{
Huda Aldosari ${ }^{1}$, Raafat Elfouly ${ }^{2}$, Reda Ammar ${ }^{3}$ and Mohammad Alsulami ${ }^{4}$ \\ $1,3,4$ University of Connecticut, \\ ${ }^{2}$ University of Rhode Island \\ ${ }^{1,3,4}\{$ huda.aldosari, reda . ammar , mohammad .alsulami@uconn . edu $\}$ \\ ${ }^{2}$ relfoulyeric.edu
}

\begin{abstract}
In this paper we propose new real time architectures for monitoring underwater oil and gas pipelines by using underwater wireless sensor network (UWSN). New monitoring architectures for underwater oil/gas pipeline inspection system combine a real time UWSN with nondestructive In Line Inspection (ILI) technology. These architecture will help in reducing or detecting the pipeline's defects such as cracks, corrosions, welds, pipeline's wall thickness ...etc by improving data transfer from the pipeline to the processor to extract useful information and deliver it to the onshore main station. Hence, decreasing delays in default detection.
\end{abstract}

\section{Introduction}

Oil and gas are considered one of the most significant energy resources in the world. The transmission of these sources between and through countries is done by pipeline that are either underwater (shallow or ultra- deep water) or underground, extending for thousands of miles and can operate for several years.

An example on of the longest gas pipeline is the one located [1] in the West-East of China, operated by PetroChina Pipelines. It extends up to $8,707 \mathrm{Km}$, passes through more than 66 cities in 10 provinces from Xianjing, China and spreads over different and several directions. It can hold 30 billion cubic meters of gas per year. An example on oil pipeline, Eastern Siberia-Pacific Ocean Oil Pipeline is one of the longest underwater pipelines which lunches from Taishet, in the Irkutsk oblast located in central Siberia, and planned to connect Eastern Siberia to the Chinese border states with 4,857 km in length ...etc.

However, such pipelines may experience some defaults, corrosions, dents, cracks, chemical defects, intentional damage and all other kinds of imperfections which can cause leaks or failures on pipelines.

G. Lee and Y. Jin (eds.), CATA 2020 (EPiC Series in Computing, vol. 69), pp. 307-316 
As a result of the consequences of pipeline leakage, several fatalities, product losses and extreme environment pollution may occur. According to [2] ProPublica, in 1986, US pipeline accidents led to around 500 deaths, over 4000 injured and $\$ 7$ billion dollars in economic loss. Pipeline accidents are still happening to this very day. [3] In January 2019, a pipeline exploded in Tlahuelilpan, Mexico, killing 137 individuals and injuring many more.

In order to avoid future accidents and decrease defects in the pipeline system, a monitoring plan should be conducted on the pipeline's infrastructures which play a crucial role in preventing or reducing the probability of any kind of hazards that may happen. In this area, several researches have been conducted to detect pipeline defaults and flaws. These researches have studied the external and internal operations of the pipelines, either by building a sensor network along with the pipeline or applying Nondestructive testing "NDT" via various of modern technologies.

Accordingly, this paper proposes using "UWSN" wireless network to create a new real time architecture. This network [4], [5], [6] developed by UCONN Research team will act as a pipeline inspection system that combines a real time UWSN sensor network paired with [7] nondestructive In Line Inspection "ILI" technology. The aim of this paper is also to propose why this architecture will reduce pipeline defaults, as this technique will improve data transfer from the pipeline to the processor by extracting useful information and delivering it to the onshore main station, and by doing so, it will decrease delays in default detection.

\section{Present Pipeline Techniques}

The security of Oil and Gas pipelines has become the focus of attention by researchers over time in order to avoid the catastrophes caused by blemish on such pipelines.

\subsection{Underwater sensor Network}

Several underwater pipeline systems have been applied and developed throughout the world. [8] Underwater pipelines are an adequate method for transferring gas, oil and water. This method has been applied by several countries because they are considered one of the most important infrastructures for economic growth and stability. According to the literature review, there are four different underwater sensors that are stated below:

\subsubsection{Underwater Wired Sensor Networks:}

This technique [9], [10] is done by using wired networks which are either made of copper or fiber optic cables connecting the pipeline's sensor devices that measure pressure, sound, motion and temperature of the pipelines and surrounding areas. There these wired sensors do not have batteries and the energy will be provided from external sources passed through the optic fiber wire. However, a downside to this technique is that any damage occurring to these wires will cause the complete or partial defect in the configuration system of the wires.

\subsubsection{Underwater Acoustic Wireless Sensor Networks:}

Unlike wired sensor pipelines, this technique is installed with underwater pipeline infrastructure communicating through acoustic sensor nodes. These nodes are used as sensors for the surrounding system and to route information from one node to the other delivering it to the control station through the closest gateway [11]. Each node is limited in its transmission meaning it can only communicate 
with its two neighbor nodes, from its left and right side, in order to transfer data. However, this method of communication may consume the sensor's power. In order to reduce the power consumption [7], a dynamic configuration for the wireless transmission range has been adopted which will provide better power management.

Thus, the node's communication range has been reduced in order to communication with one neighbor node from each side thus reducing power consumption. If one of the node's neighbor faced damage, the node's range will automatically expand to the next neighbor in order to maintain data transfer and deliver it to the gateway [5].

\subsubsection{RF (Radio Frequency) Wireless Sensor Networks:}

Since acoustic wireless sensor has suffered some major problems due to the limited bandwidth available among the nodes which can greatly increase delays and power consumption in data transmission [12]. Radio frequency wireless technique has been developed to monitor under water pipelines. This technique operates through sensor nodes that are directly connected to its surface gateway. The data transmission between the nodes is done through their direct gateway, the node and the gateway are connected through wires. As a result, the nodes will be communication through radio frequency channels. Even though this technique has many advantages over other techniques such as faster communication, it has two major disadvantages. First, the wires may be damaged easily by any passing ships or moving objects. Second, the pipelines can be easily found from the gateway and thus may be sabotaged.

\subsubsection{Integrated Wired/ Acoustic Wireless Sensor Networks:}

This technique has integrated the wired and acoustic sensor networks. [13] Nodes are now connected through wired and acoustic sensors. However, wires are faster and transfer power between nodes. If any damage occurs to the wire then the acoustic sensor will be activated instead of the total network shutdown.

\subsubsection{Integrated Wired/ Wireless Sensor Networks:}

In this technique [7], radio frequency channels are used instead of acoustic communication channels. Therefore, the communication between the nodes is done through the RF gateway. A radio frequency gateway is released and therefore activated when there is a default such as a cut or fault in the wire connecting the nodes. Therefore, the communication between the nodes is done through the RF gateway. This method provides more security to the pipelines.

\subsection{Non-destructive technologies:}

\subsubsection{Remotely Operated Vehicle (ROV):}

As the name suggests, remotely operated vehicles (ROV) are underwater vehicles that are remotely controlled from the surface. There is a cable that connects the surface platform to the vehicle that provides it with power. There are three types of ROVs, bottom-crawling towed, bottom-crawling tethered, and free-swimming tethered (which is the most common type). Although free-swimming is the most common type, bottom-crawling are the ones primarily used in pipeline and cable work [14]. These vehicles go down to the bottom of the ocean, and maneuver on wheels. The purpose of ROVs is to collect video footage of our oceans that fuel our scientific knowledge. 


\subsubsection{Autonomous Underwater Vehicle (AUV):}

Autonomous underwater vehicles are robotic machinery that navigate the deep parts of the oceans and other underwater areas that are unsafe for humans. AUVs rely on an onboard computer system that functions in decision making. AUVs have been gaining popularity for work that involves deep underwater exploration as they last for long hours, and unlike ROVs, they are not tethered to a wire that's connected to the surface. AUVs navigate autonomously, using their own coded algorithm to assess its surroundings [13]. It has been a challenge to devise a computer device that navigates autonomously and explore hostile territories, but with the recent advancements of AUVs hardware material and software, they have become very practical and reliable.

\subsubsection{In Line Inspection (ILI)}

In-line inspection involves the use of pipelines in the gas and oil industry. As there is always a risk of corrosion, pipe leakage, and damage to be done underwater, there has to be a thorough inspection that periodically detects these dangers as to ensure a safer work environment. However, due largely to interruptions and failures, in-line inspections have become a commonly employed evaluation method to determine risks and failures. The ILI [15] is inserted into the pipeline every few years to ensure the integrity of such pipelines. The ILI inserted at the pipeline launch station where it flows along with oil or gas to detect any kind of defects through its Ultrasonic, Magnetic Flux Leakage, ... sensors. The inspection data is released upon arrival at its destination.

\section{Proposed architecture}

Since oil and gas are one of the leading resources that provide energy and increase economics for a country, it is crucial to maintain these resources safety from any critical defects. Most of underwater oil and gas pipelines have started their operations for up to tens to hundreds of years. Thus, ageing pipelines usually suffer from some corrosions or defects which require an immediate response from an onshore control station to avert any catastrophe that may happen.

Here, new architectures for underwater oil and gas pipeline sensor network is proposed which provides a significant improvement in end-to-end delay, and will be near real time. The main idea of this architecture is built based on UWSN underwater sensor network which was developed by UCONN. [4], [5], [6] Such a system is used to serve numerus monitoring and detecting applications such as 3-D mapping, fish detection and classification, surveillance, intrusion detection, and military applications that takes place underwater. According to [8] the designed underwater sensor network has a capability to sense, communicate, and forward data form sensor nodes to an offshore control station with less power consumption. The deployment of this architecture [6] has four main components as the following: First, a central computer used to process data, extract valuable information, and then transmit it to the gateway. Second, sensor nodes are used to sense data in its region. Third, a master node to collect data from different sensor nodes and send it to the central computer. The last component is a gateway which receives information from the central computer or master nodes and send it to the main station.

Some modifications have been made on this architecture to fit on underwater pipelines. Instead of having an acoustic wireless network for the whole system, two communication methods have been operated to sense, communicate and transfer data between nodes along with the pipeline. Nodes are now connected through wired and acoustic sensors. There are however some limitations [16] of acoustic wireless communication, such as propagation delay, low bandwidth, and a high energy requirement. 
However, wire sensors are faster and transfer power between nodes. If any damage occurs to the wire then the acoustic sensor will be activated instead of the total network shutdown. In addition, the communication between a master node and processor node is done through acoustic wave to avoid any damage that may happen to a wire. The components of a such system are as following:

- Processor node: the main function of the processor node is to process the data and extract the valuable information and then transmit it to the control station via multi relay nodes. It is cheaper than a computer and has a capability to process a high volume of data. The type of processor depends on the volume of data that is acquired from sensors node or ILI.

- Master node: it locates on each pipeline segment of a pipeline. It operates as sensing, collecting, and transferring its data and all nodes located on its segment or neighbor segments' data to a dedicated processor.

- Sensor: it has ability to sense the pipeline surrounding area and transfer its data to a master node through single or multi-hops

- Relay node: to boost the acoustic wave when the distance between two communicating nodes is more than $200 \mathrm{~m}$.

- Gateway: the main purpose of this node is to receive data from a hyper-processing node via relay node and transmit it to a control station through wireless communication.

\section{Three different architectures}

We propose three architectures for inspection purpose, [13], [17] there are a plethora of In line inspection tools (smart pigs) available currently in the market for examining the extensive underwater pipelines. Each of them is capable to detect one or more type/s of pipeline's defects either externally or internally or both depending on the technology used. [18] Recently, different inspection technologies have been combined into one in line inspection tool which makes it possible to detect different types of defects/flaws such as cracks, corrosions, or wall thickness ...etc. In addition, it would provide its type and size at the same time. However, the data will be analyzed when pigs arrive to the destination. To achieve a real time system, we proposed a new underwater wired/acoustic sensor network for oil and gas pipeline with three different architectures. The network that this paper is proposing is a system that can be used without communication with ILI, or with communication.

\subsection{Architecture one:}

In this architecture, the system consists of one master node for each pipeline's segment. Each master node is capable to sense, collect the right-side pipeline's segment data from its sensors and then transmit it to a dedicated processor node. The processor node processes such data and then delivers it to a gateway via one or multiple relay nodes if the distance to the gateway is more than $200 \mathrm{~m}$. To illustrate this architecture, figure (1) shows the main components: 


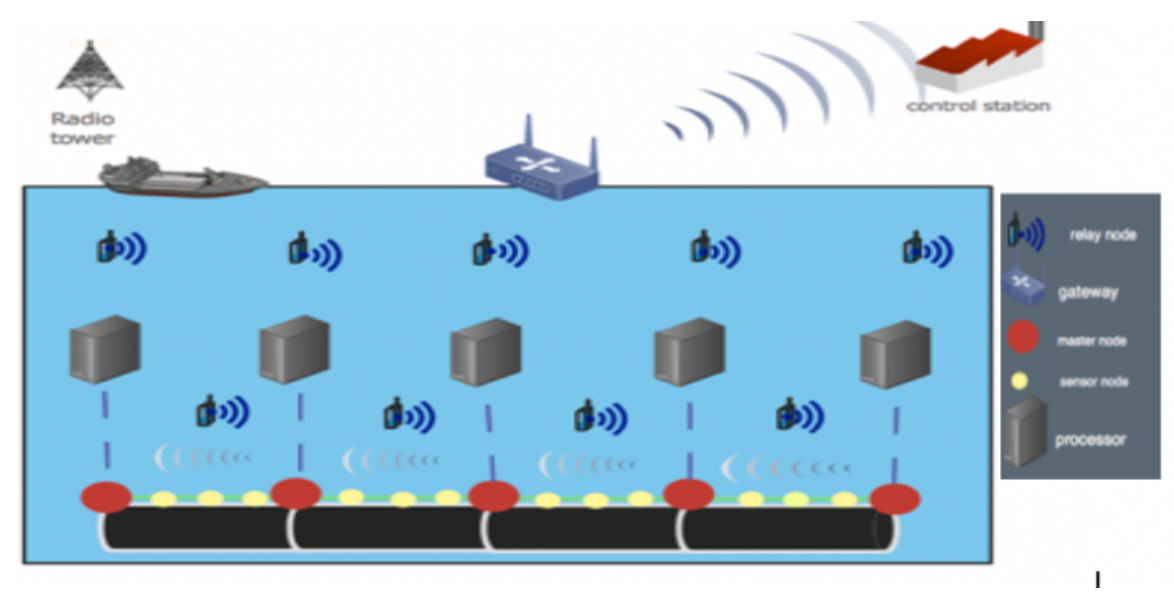

Figure 1: Architecture one of a real time underwater sensor network for oil and gas pipelines.

\subsection{Architecture two:}

This architecture is similar to architecture $\# 1$, however, instead of a master node to collect data from only the right-side pipeline's segment, it collects data from both pipeline's segments (left and right). Figure (2) demonstrates this architecture.

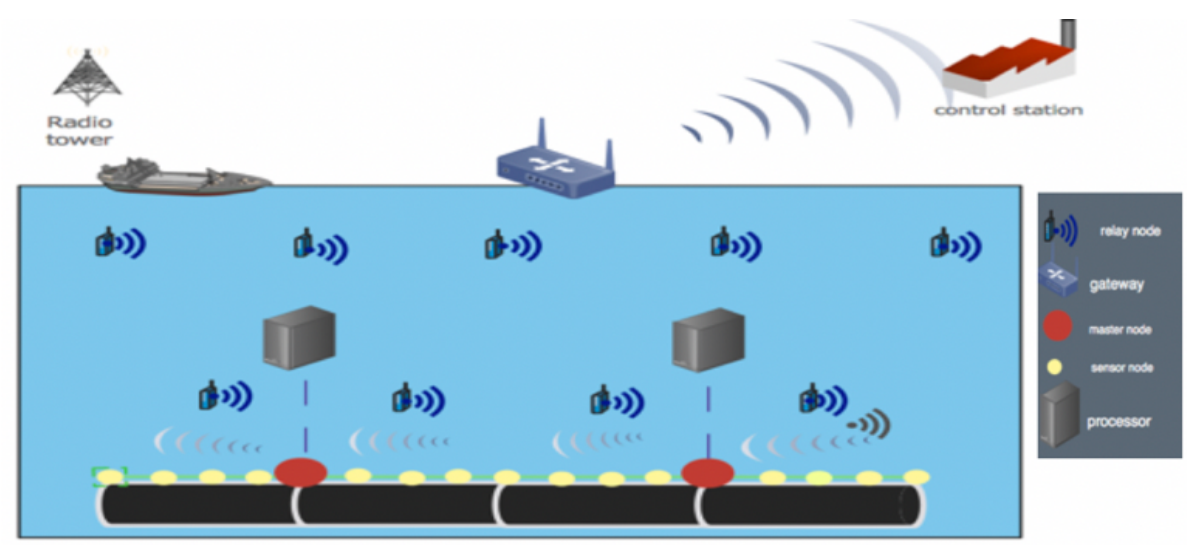

Figure 2:Architecture two of a real time underwater sensor network for oil and gas pipelines.

\subsection{Architecture three:}

In this architecture, each pipeline' segment has one master node located on the left-side of the segment. Those master nodes sense and collect data from the right-side segment sensors, and then transmit data to the processor node via multi relay nodes. The processor nodes have the capability to process high a volume of data and extract the useful information and send it to a control station through a gateway. Figure (3) shows this architecture in details. 


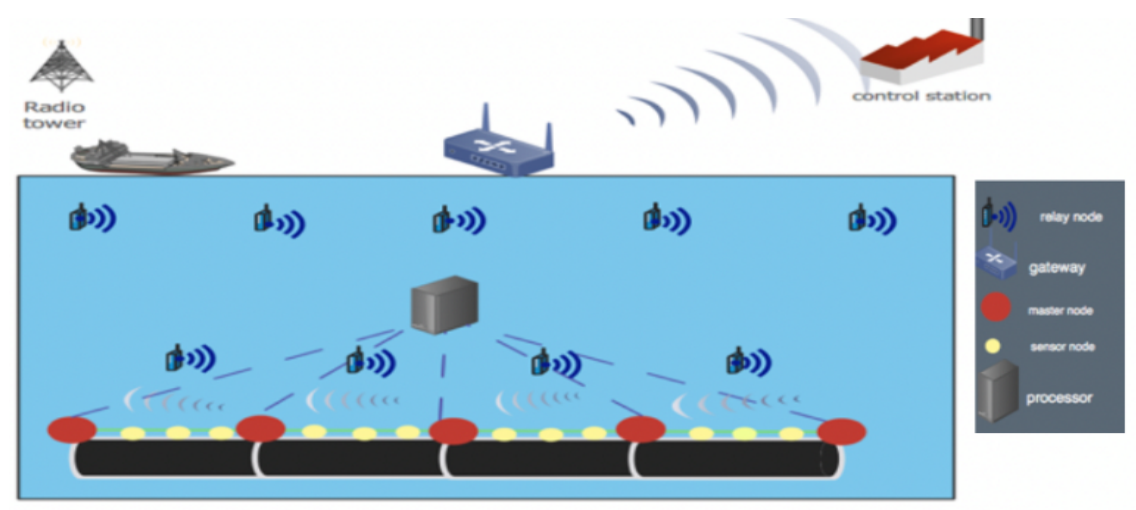

Figure 3:Architecture three of a real time underwater sensor network for oil and gas pipelines.

\section{Integrating UWSN with ILI Communication scenarios:}

We studied three communication scenarios for each of the architectures explained above. Figure $(4,5,6)$ shows that each of the architectures can apply to each of these scenarios based on needs. Therefore, we have nine different cases in total.

Scenario 1: Here, the ILI tool communicates with only a master node once a defect has been detected. If no flaws are appeared, the ILI continue moving to the next pipeline's segment.

Scenario 2: the ILI tools communicates with either a sensor node or a master node that located closest to the detected defect. Two cases may happen:

- In case the defect occurs close to a sensor node, the ILI sends the data to that sensor node, then the sensor node sends the data to a master node via single or multi- hop.

- If a defect occurs between a sensor node and a master node, the ILI tool directly sends the data to the master node.

Scenario 3: the ILI tools communicates with either a sensor node or a master node that located closest to the detected defect. If the defect occurred close to a sensor node, the ILI will send the data to that node, then this sensor node becomes a master node and sends the data to the processor node directly.

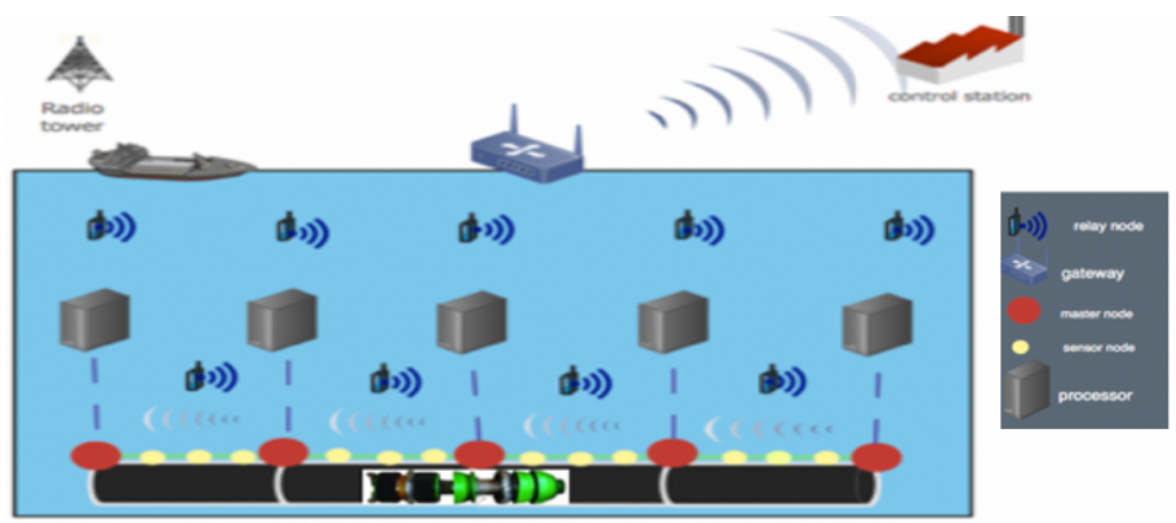

Figure 4: Architecture one applied different scenarios 


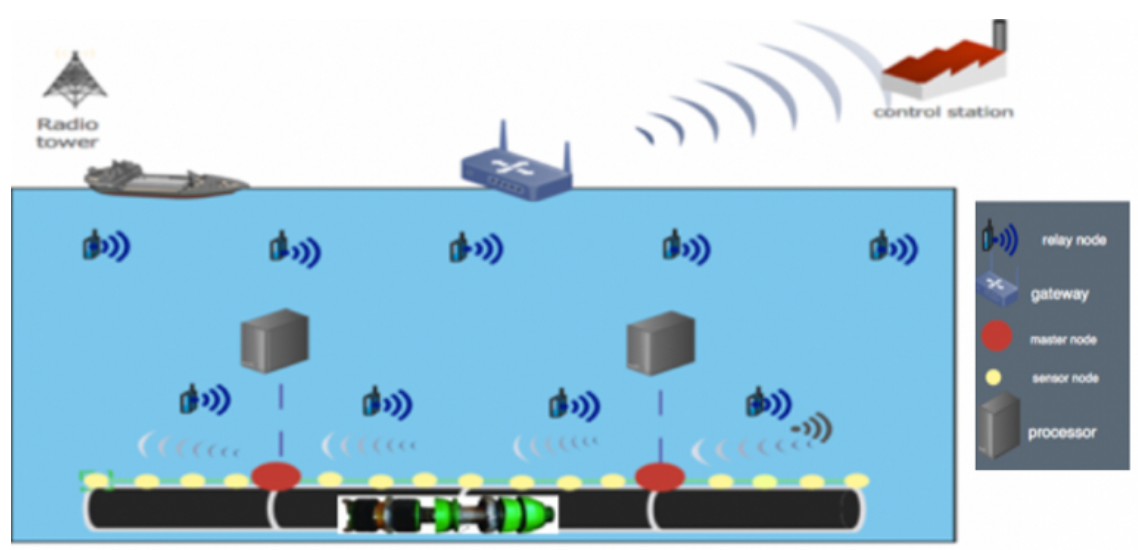

Figure 5:Architecture two applied different scenarios

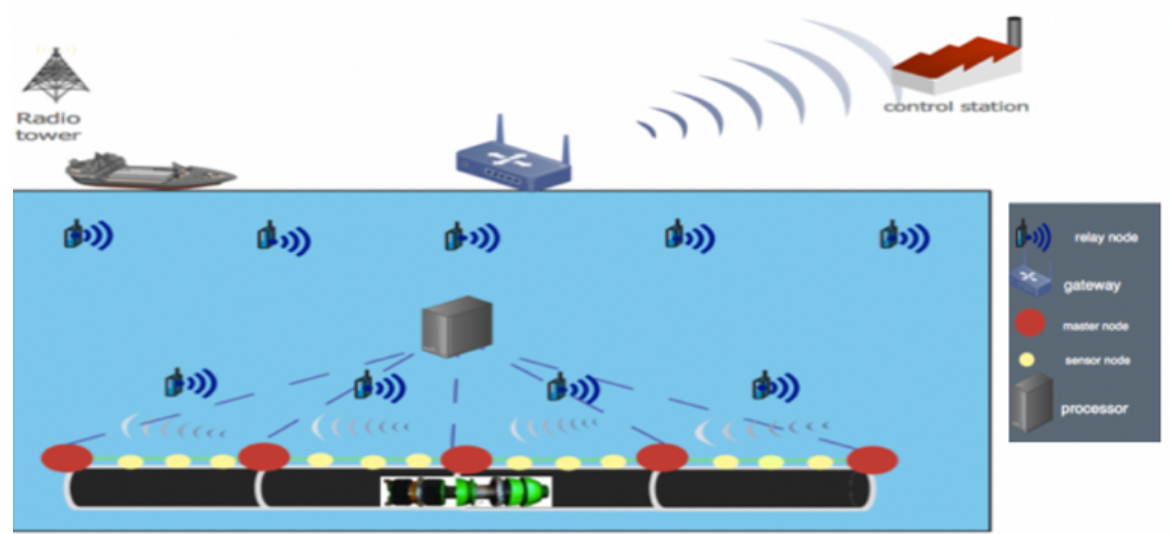

Figure 6:Architecture three applied different scenarios

\section{Result}

For all mentioned architectures, two communications methods are used for more network reliability and speed up the network performance. The main communication method used is wired communication which connects all sensors node and master nodes in a linear way. If any failure occurs on the optical cable, acoustic waves will be activated instead of shutdown the whole system but that causes reduction in the system performance. However, the communication between all master nodes and processors and then to gateways is acoustic waves.

With the proposed architectures, we can divide the system into two parts. One is the wired/Acoustic network that located on the pipeline. The other is the acoustic communication between master nodes, processors and gateways. The system performance for the second part, which is the acoustic communication from master nodes to gateways via processors, has been calculated in [6] and showed improvement in end to end delay. Figure (6) illustrates the performance for the second part of the system using different processing ratio and with depth $1000 \mathrm{~m}$ and data size $1.5 \mathrm{MB}$. 


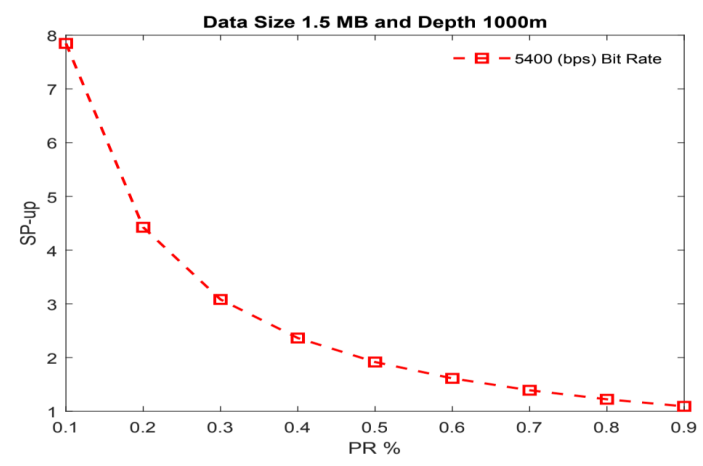

Figure 7: the speed up for the second part of the system

Since the performance of wired communication (optical fiber cable) is very high and the new architecture that has hyper-processors to extract valuable information shows that reduction in overall transmission time, any kinds of pipeline defects will be transferred immediately from ILI to an onshore control station through UWSN.

\section{CONCLUSION}

Oil and gas are conceived as one of the most essential assets on the planet. The transmission of these sources between and through nations is finished by pipelines that are either submerged (shallow or ultraprofound water) or underground. They can stretch out for a great number of miles and can work for quite a long while, if not decades. A large portion of submerged oil and gas pipelines have begun their tasks for up to many years. Accordingly, those maturing pipelines typically experience the ill effects of certain imperfections which require a prompt reaction from a coastal control station to deflect any calamities that may occur. The security of Oil and Gas pipelines has turned into the focal point of consideration by scientists in decades so as to maintain a strategic distance from the cataclysmic brought about by flaw on such pipelines. The goal of this work is to develop a real time inspection system for underwater oil and gas pipeline with minimum end to end delay. As a result, this will decrease number of pipeline defects but not prevent it. Thus, fewer defaults by improving information flow from the pipeline to the processor and conveying it to the inland fundamental station. However, some investigations regarding data size and rate are still in progress. [19] A huge amount of data either unstructured or multi-structured data is generated continuously by sensors and/or ILI tools. [20] Those data are considered as big data and may be up to 5 exabyte in two days which needs to high processing and transmission rate. However, the available number of processing units are limited comparing to the data. The inspection data can be different in size, formats such as text, video, or audio. According to [21] $90 \%$ of generated data is unstructured. all above data are not final and still need work to be done

\section{REFERENCES}

[1] T. Husseini, "The world's longest pipelines in the oil and gas industry," Offshore Technology | Oil and Gas News and Market Analysis, 14-Sep-2018. [Online]. Available: https://www.offshoretechnology.com/features/worlds-longest-pipelines/. [Accessed: 10-Oct-2019].

[2] L. V. Groeger, "Pipelines Explained: How Safe are America's 2.5 Million Miles of Pipelines?," ProPublica, 09-Mar-2019. [Online]. Available: 
https://www.propublica.org/article/pipelines-explained-how-safe-are-americas-2.5-million-milesof-pipelines. [Accessed: 03-Oct-2019].

[3] Bloomberg.com. [Online]. Available: https://www.bloomberg.com/news/features/2019-06-26/agas-heist-gone-wrong-an-explosion-and-137-deaths-in-mexico. [Accessed: 10-Oct-2019].

[4] S. Ibrahim, J.-H. Cui, and R. Ammar, "Surface-Level Gateway Deployment for Underwater Sensor Networks," MILCOM 2007 - IEEE Military Communications Conference, 2007.

[5] A. Alharbi, H. Alhumyani, S. Tolba, R. Ammar, and Jun-Hong, "Underwater Sensing and Processing Networks (USPN)," 2014 IEEE Symposium on Computers and Communications (ISCC), 2014.

[6] H. Albarakati, A. Amamra, R. Elfouly, and R. Ammar, "Reconfigurable underwater embedded systems architectures," 2017 IEEE Symposium on Computers and Communications (ISCC), 2017.

[7] H. Ghannadrezaii and J.-F. Bousquet, "Maximizing network coverage in a multichannel shortrange underwater acoustic sensor network," Computer Networks, vol. 160, pp. 1-10, 2019.

[8] W.-M. Tian, "Integrated method for the detection and location of underwater pipelines," Applied Acoustics, vol. 69, no. 5, pp. 387-398, 2008.

[9] A. Carrillo, E. Gonzalez, A. Rosas, and A. Marquez, "New distributed optical sensor for detection and localization of liquid leaks," Sensors and Actuators A: Physical, vol. 99, no. 3, pp. 229-235, 2002.

[10] W.-W. Lin, "Novel distributed fiber optic leak detection system," Optical Engineering, vol. 43, no. 2, p. 278, Jan. 2004.

[11] J.-H. Cui, J. Kong, M. Gerla, and S. Zhou, "The challenges of building scalable mobile underwater wireless sensor networks for aquatic applications," IEEE Network, vol. 20, no. 3, pp. $12-18,2006$.

[12] J. G. Proakis, J. A. Rice, E. M. Sozer, and M. Stojanovic, "Shallow-Water Acoustic Networks*," Wiley Encyclopedia of Telecommunications, 2003.

[13] A. Sahoo, S. K. Dwivedy, and P. Robi, "Advancements in the field of autonomous underwater vehicle," Ocean Engineering, vol. 181, pp. 145-160, 2019.

[14] K. Shepherd, "Remotely Operated Vehicles (ROVs)," Encyclopedia of Ocean Sciences, pp. 742 747, 2001.

[15] Q. Liang, W. Zhu, W. Sun, Z. Yu, Y. Wang, and D. Zhang, "In-line inspection solution for codes on complex backgrounds for the plastic container industry," Measurement, vol. 148, p. 106965, 2019.

[16] F. Núñez, Y. Wang, D. Grasing, S. Desai, G. Cakiades, and F. J. Doyle, "Pulse-coupled time synchronization for distributed acoustic event detection using wireless sensor networks," Control Engineering Practice, vol. 60, pp. 106-117, 2017.

[17] P. I. Macreadie, D. L. Mclean, P. G. Thomson, J. C. Partridge, D. O. Jones, A. R. Gates, M. C. Benfield, S. P. Collin, D. J. Booth, L. L. Smith, E. Techera, D. Skropeta, T. Horton, C. Pattiaratchi, T. Bond, and A. M. Fowler, "Eyes in the sea: Unlocking the mysteries of the ocean using industrial, remotely operated vehicles (ROVs)," Science of The Total Environment, vol. 634, pp. 1077-1091, 2018.

[18] "The Advantages and Disadvantages of In-Line Inspection Tools," Inspectioneering. [Online]. Available: https://inspectioneering.com/journal/2017-04-27/6416/a-history-of-in-line-inspectiontools. [Accessed: 01-Oct-2019].

[19] M. Mohammadpoor and F. Torabi, "Big Data analytics in oil and gas industry: An emerging trend," Petroleum, 2018.

[20] M. S. Sumbal, E. Tsui, and E. W. See-To, "Interrelationship between big data and knowledge management: an exploratory study in the oil and gas sector," Journal of Knowledge Management, vol. 21, no. 1, pp. 180-196, 2017.

[21] Ishwarappa and J. Anuradha, "A Brief Introduction on Big Data 5Vs Characteristics and Hadoop Technology," Procedia Computer Science, vol. 48, pp. 319-324, 2015. 